\title{
Comparison of the efficacy of irrigation with epinephrine or tranexamic acid on visual clarity during arthroscopic rotator cuff repair: A double-blind, randomized-controlled study
}

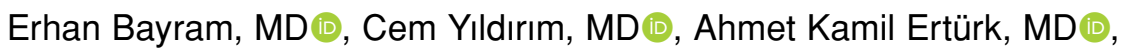 \\ Murat Yılmaz, MD D, Doğan Atlıhan, MD D \\ Department of Orthopedics and Traumatology, University of Health Sciences, Haseki Training and Research Hospital, Istanbul, Turkey
}

Over the past decade, arthroscopic repair has been recognized as an important treatment option for rotator cuff tears. Sufficient visualization is of paramount importance for the arthroscopic repair to be efficiently and effectively performed. Intra-articular bleeding is one of the main factors which compromises the visual clarity in arthroscopic procedures. ${ }^{[1,2]}$ Therefore, several hemostatic techniques, including hypotensive anesthesia, electrocauterization, and use of pressure- or flow-controlled pumps, are utilized to prevent intraoperative bleeding and to obtain sufficient visualization during the arthroscopic surgical procedures..$^{[3-5]}$

Recently, a number of clinical trials have supported the use of irrigation fluid with

Received: August 07, 2020

Accepted: November 18, 2020

Published online: January 06, 2021

Correspondence: Erhan Bayram, MD. SBÜ Haseki Eğitim ve Araştırma Hastanesi Ortopedi ve Travmatoloji Kliniği, 34096 Fatih, İstanbul, Türkiye.

E-mail: bayerhan@yahoo.com

Doi: 10.5606/ehc. 2021.78393

Citation: Bayram E, Yıldırım C, Ertürk AK, Yılmaz M, Atlıhan D. Comparison of the efficacy of irrigation with epinephrine or tranexamic acid on visual clarity during arthroscopic rotator cuff repair: A double-blind, randomized-controlled study. Jt Dis Relat Surg 2021;32(1):115-121.

(02021 All right reserved by the Turkish Joint Diseases Foundation

This is an open access article under the terms of the Creative Commons Attribution-NonCommercial License, which permits use, distribution and reproduction in any medium, provided the original work is properly cited and is not used for commercial purposes (http://creativecommons.org/licenses/by-nc/4.0/).

\section{ABSTRACT}

Objectives: This study aims to investigate whether there was a difference between epinephrine (EPN) and tranexamic acid (TXA) in providing visual clarity during arthroscopic rotator cuff tear repair.

Patients and methods: This double-blind, prospective, randomized-controlled clinical study included a total of 90 patients (42 males, 48 females; mean age: 55.6 \pm 8.3 years; range, 18 to 69 years) in whom either EPN (EPN group, $n=47$ ) or TXA-diluted irrigation solutions (TXA group, $n=43$ ) were used during rotator cuff tear arthroscopy between December 2017 and November 2019. Arthroscopy was performed using irrigation fluid containing $0.33 \mathrm{mg}$ of EPN per $1 \mathrm{~L}$ of saline in the EPN group and $0.42 \mathrm{mg}$ of TXA per $1 \mathrm{~L}$ of saline in the TXA group. All procedures were performed by two specialized shoulder surgeons. Visual clarity (primary endpoint) was rated by the operating surgeon using the Visual Analog Scale (VAS) in the immediate postoperative period. Secondary endpoints included total operating time (TOT), potential thrombotic or thromboembolic side effects, mean arterial pressure (MAP), and total amount of irrigation fluid used.

Results: There was no significant difference in the surgeon ratedVAS scores between the groups. The mean VAS score was 7.6 \pm 1.62 (range, 4 to 10) in the EPN group and 7.1 \pm 1.74 (range, 3 to 10) in the TXA group $(\mathrm{p}=0.59)$. No cardiac, thrombotic, or thromboembolic complications were observed in any of the groups.

Conclusion: Adding TXA to the irrigation fluid during the arthroscopic rotator cuff repair may provide similar visual quality to the EPN, as measured by VAS.

Keywords: Cuff tear repair, epinephrine, image quality, irrigation, shoulder arthroscopy, tranexamic acid; visual clarity.

epinephrine (EPN) to decrease blood loss and improve arthroscopic visualization..$^{[2,6,7]}$ Furthermore, more recent studies have shown that intravenous or topical administration of tranexamic acid (TXA), which is an antifibrinolytic agent, is effective in reducing intraoperative 
bleeding during shoulder and knee replacement surgery, ${ }^{[8,9]}$ improving visual clarity in arthroscopic shoulder surgery, ${ }^{[10]}$ and reducing hemarthrosis following the arthroscopic anterior cruciate ligament reconstruction. ${ }^{[11]}$ Nonetheless, to the best of our knowledge, no study has investigated the effect of TXA versus EPN yet, when used in irrigation solution on visual clarity during arthroscopic rotator cuff repair.

In the present study, we hypothesized that the addition of TXA to the irrigation fluid during rotator cuff tear arthroscopy might be superior to EPN in providing visual clarity. We, therefore, aimed to investigate whether there was a difference between the addition of EPN and the addition of TXA to the irrigation fluid in providing visual clarity during arthroscopic rotator cuff tear repair.

\section{PATIENTS AND METHODS}

\section{Study design and study population}

This double-blind, prospective, randomizedcontrolled clinical study included patients in whom either EPN (EPN group, $\mathrm{n}=47$ ) or TXA-diluted irrigation solutions (TXA group, $n=43$ ) were used during rotator cuff tear arthroscopy between December 2017 and November 2019. All patients were informed about the nature of the study and a written informed consent was obtained. The study protocol was approved by the Haseki Training and Research Hospital Ethics Committee (Approval date/no: 2017-580). The study was conducted in accordance with the principles of the Declaration of Helsinki. This study is registered to ClinicalTrials. gov (NCT04628676).
The patients who fulfilled the inclusion criteria (Table I) were randomly assigned to one of two groups: EPN and TXA. Randomization was undertaken using a computer-generated randomization allocation table produced by an anesthesiologist who was not involved in the recruitment phase. The irrigation solution was prepared by a nurse in another room according to the patient group. Both the surgeons and patients were blinded as to which group they were assigned.

Overall, 50 patients underwent arthroscopic double-row rotator cuff repair using an irrigation fluid with EPN (Group EPN), and 50 patients underwent arthroscopic double-row rotator cuff repair using an irrigation fluid with TXA (Group TXA). In patients receiving anticoagulants, therapy was discontinued five days before shoulder arthroscopy. Arthroscopy was performed using an irrigation fluid containing $0.33 \mathrm{mg}$ of EPN per $1 \mathrm{~L}$ of saline in the EPN group and $0.42 \mathrm{~g}$ of TXA per $1 \mathrm{~L}$ of saline in the TXA group. After excluding 10 patients $(\mathrm{n}=6$ uncontrolled hypertension and $\mathrm{n}=4$ an unrepairable rotator cuff tear during the operation), the study was completed with 90 patients (42 males, 48 females; mean age: $55.6 \pm 8.3$ years; range, 18 to 69 years). The study flow chart is shown in Figure 1.

\section{Study endpoints and definitions}

The primary endpoint of this study was the visual clarity rated by the operating surgeons using the Visual Analog Scale (VAS) in the immediate postoperative period, as previously described by Avery et al. ${ }^{[6]}$ The VAS scores range from 1 to 10, with a score of 1 indicating complete lack of visualization

\begin{tabular}{|c|c|}
\hline \multicolumn{2}{|c|}{$\begin{array}{l}\text { TABLE I } \\
\text { Eligibility criteria of study population }\end{array}$} \\
\hline Inclusion criteria & Exclusion criteria \\
\hline $\begin{array}{l}\text { - Age }>18 \text { years } \\
\text { - } \text { ASA }^{*} \text { score: } 1-2 \\
\text { - Undergoing a full arthroscopic double-row repair due to } \\
\text { - } \text { Being willing to participate the study }\end{array}$ & $\begin{array}{l}\text { - } \text { Age }<18 \text { years } \\
\text { - } A S A \geq 3 \\
\text { - Concomitant cardiac diseases } \\
\text { - Concomitant bleeding disorder or coagulopathy } \\
\text { - Diabetes mellitus } \\
\text { - Being pregnant } \\
\text { - Use of contraceptive pills } \\
\text { - A history of previous shoulder surgery } \\
\text { - Uncontrolled hypertension during the operation }(>180 / 110 \mathrm{mmHg}) \\
\text { - Unrepairable rotator cuff tears } \\
\text { - Being unwilling to participate the study }\end{array}$ \\
\hline
\end{tabular}



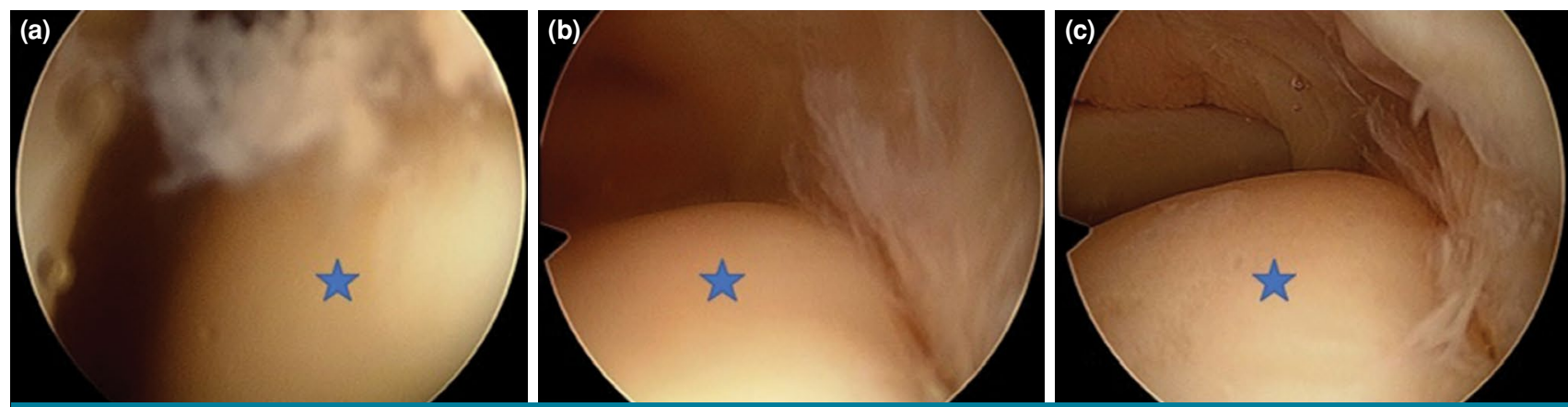

FIGURE 1. Representative images of VAS scores. Representative examples of VAS scores as seen by the posterior viewing portal in the glenohumeral joint. VAS scores are illustrated for values of 3 (a), 7 (b), 10 (c). Stars denote humeral head.

VAS: Visual Analog Scale.

and a score of 10 indicating the best visualization possible. Figure 2 demonstrates representative samples of VAS scores.

Secondary endpoints included total operating time (TOT), mean arterial pressure (MAP), total amount of irrigation fluid used intraoperatively, the need for surgeon-demanded intravenous blood pressure therapy, and potential adverse cardiac, thrombotic or thromboembolic effects.

The TOT was defined as the period in min from the time the arthroscope was inserted into the glenohumeral joint until the termination of the arthroscopic procedure. Adverse cardiac effects were defined as electrocardiographic abnormalities or arrhythmias during surgery. The aforementioned parameters were noted on the patient sheet by the anesthesiologist. All patients were also clinically monitored for developing thrombotic or thromboembolic events during the postoperative hospitalization.

\section{Surgical technique}

With the patient in the beach chair position, all procedures were performed under general anesthesia by two orthopedic surgeons who specialized in shoulder arthroscopy. All operations were standardized, and both surgeons executed similar operative steps in the same sequence, regardless of the patient allocation to the EPN or TXA groups. All rotator cuff repairs were executed by the transosseous-equivalent double-row technique. The size of the tear was measured in $\mathrm{cm}$ at the time of surgery. Based on the tear size, one or two metal anchors (Smith \& Nephew Ltd., London, UK) were applied for the medial row. After sutures were tied

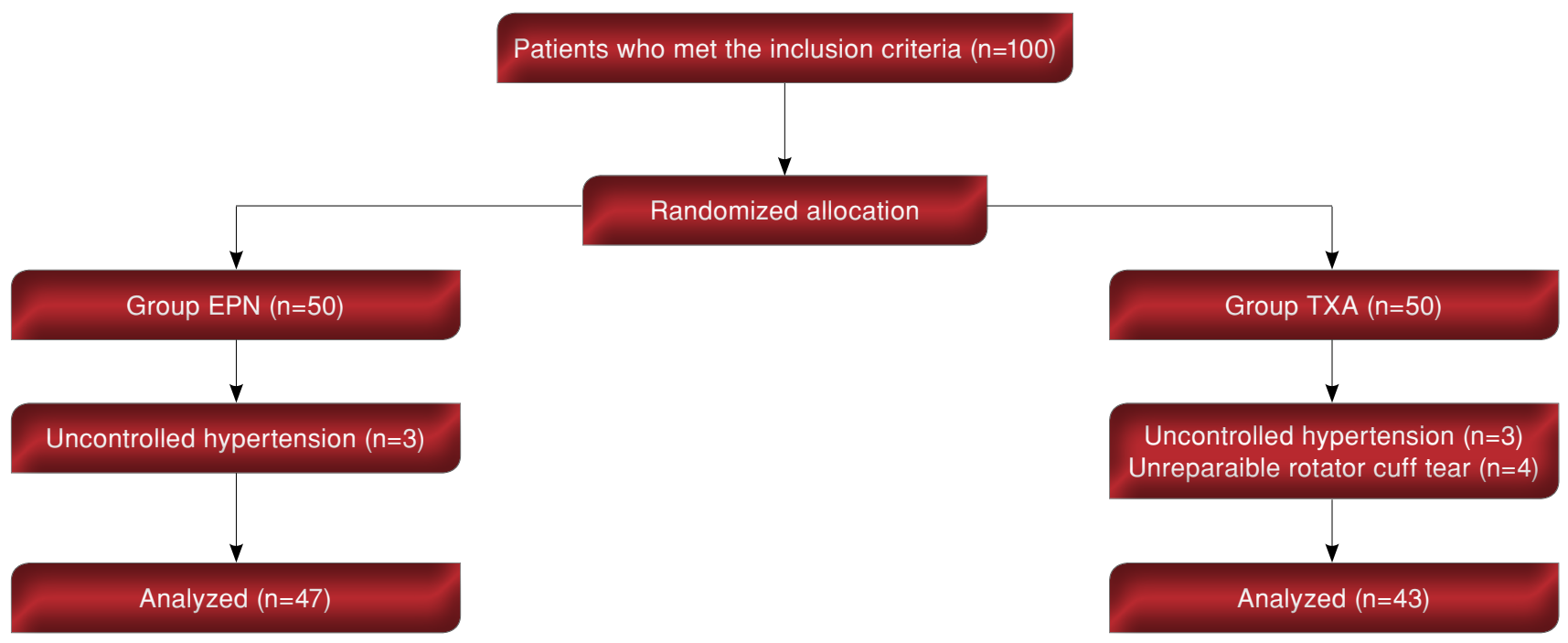




\begin{tabular}{|c|c|c|c|c|c|c|c|}
\hline \multicolumn{8}{|c|}{$\begin{array}{c}\text { TABLE II } \\
\text { d clinical characteris }\end{array}$} \\
\hline & \multicolumn{3}{|c|}{ Group EPN $(n=47)$} & \multicolumn{3}{|c|}{ Group TXA $(n=43)$} & \multirow[b]{2}{*}{$p$} \\
\hline & $n$ & Mean \pm SD & Range & $\mathrm{n}$ & Mean $\pm S D$ & Range & \\
\hline Age (year) & & $57 \pm 8.3$ & 18 to 68 & & $54 \pm 8.1$ & 19 to 66 & 0.142 \\
\hline Sex & & & & & & & 0.79 \\
\hline Male & 22 & & & 20 & & & \\
\hline Female & 25 & & & 23 & & & \\
\hline Body mass index $\left(\mathrm{kg} / \mathrm{m}^{2}\right)$ & & $31 \pm 5.4$ & $26-37$ & & $30 \pm 7.3$ & $24-38$ & 0.86 \\
\hline Tear size $(\mathrm{cm})$ & & $1.9 \pm 1.0$ & $0.5-4$ & & $2.0 \pm 0.9$ & $0.5-4$ & 0.79 \\
\hline Number of anchors & & $2.2 \pm 0.5$ & $1-3$ & & $2.2 \pm 0.5$ & $1-3$ & 0.81 \\
\hline
\end{tabular}

in the medial row, the lateral row was fixed using one or two knotless anchors (FOOTPRINT ${ }^{\circledR}$; Smith \& Nephew Ltd., London, UK).

\section{Statistical analysis}

Study power analysis and sample size calculation were performed using the G*Power version 3.1.6 software (Heinrich-Heine-Universität Düsseldorf, Düsseldorf, Germany). For an effect size of 0.5 , and alpha error probability of 0.05 , minimum 50 patients per group were required to ensure $80 \%$ study power. Statistical analysis was performed using the IBM SPSS version 20.0 software (IBM Corp., Armonk, NY, USA). Descriptive data were expressed in mean \pm standard deviation (SD), median (min-max) or number and frequency. Inter-group comparisons were performed using the Student's t-test or Mann-Whitney U test for continuous variables and using the Pearson chi-square test, Fisher's exact test, or Fisher-Freeman-Halton exact test for categorical variables. A $p$ value of $<0.05$ was considered statistically significant.

\section{RESULTS}

Baseline demographic and clinical characteristics of the patients are shown in Table II. There was no significant difference in the demographic and clinical data between the groups $(\mathrm{p}>0.05)$.

In addition, there was no significant difference in the surgeon rated-VAS scores between the groups. The mean VAS score was $7.6 \pm 1.62$ (range, 4 to 10 ) in the EPN group and 7.1 \pm 1.74 (range, 3 to 10 ) in the TXA group ( $\mathrm{p}=0.59)$ (Table III).

\begin{tabular}{|c|c|c|c|c|c|}
\hline \multicolumn{6}{|c|}{$\begin{array}{l}\text { TABLE III } \\
\text { res of patient groups }\end{array}$} \\
\hline \multirow[b]{2}{*}{ Variable } & \multicolumn{2}{|c|}{ Group EPN $(n=47)$} & \multicolumn{2}{|c|}{ Group TXA $(n=43)$} & \multirow[b]{2}{*}{$p$} \\
\hline & Mean $\pm S D$ & Range & Mean \pm SD & Range & \\
\hline VAS score & $7.6 \pm 1.62$ & $4-10$ & $7.1 \pm 1.74$ & $3-10$ & 0.59 \\
\hline
\end{tabular}

\begin{tabular}{|c|c|c|c|c|c|c|c|c|c|}
\hline \multirow[b]{3}{*}{ Variables } & \multicolumn{8}{|c|}{$\begin{array}{c}\text { TABLE IV } \\
\text { Comparison of secondary endpoints }\end{array}$} & \multirow[b]{3}{*}{$p$} \\
\hline & \multicolumn{4}{|c|}{ Group EPN $(n=47)$} & \multicolumn{4}{|c|}{ Group TXA $(n=43)$} & \\
\hline & $\mathrm{n}$ & $\%$ & Mean $\pm S D$ & Range & $\mathrm{n}$ & $\%$ & Mean $\pm S D$ & Range & \\
\hline Total operating time (min) & & & $103.4 \pm 21.1$ & $18-68$ & & & $105.7 \pm 23.5$ & $19-66$ & 0.26 \\
\hline Average MAP (mmHg) & & & $85.4 \pm 11.3$ & & & & $83.3 \pm 10.9$ & & 0.512 \\
\hline Amount of fluid used (min) & & & $8.5 \pm 5.4$ & & & & $8.1 \pm 4.6$ & & 0.76 \\
\hline Blood pressure therapy & 6 & $12 \%$ & & & 4 & 9 & & & 0.45 \\
\hline
\end{tabular}


Also, there were no significant differences in any of the secondary endpoints between the two groups (Table IV). No cardiac, thrombotic, or thromboembolic complications were observed in any of the groups.

\section{DISCUSSION}

In the present study, we investigated whether there was a difference between EPN and TXA in providing visual clarity during arthroscopic rotator cuff tear repair. The main finding of the present study is that adding TXA to the irrigation fluid during arthroscopic rotator cuff repair provided similar visual quality to EPN, as measured by the VAS.

High-quality vision is one of the essential factors for the safety and success of arthroscopic surgery. However, intra-articular bleeding during arthroscopic procedures can complicate surgery due to loss of vision, particularly in the shoulder joint. Accordingly, recent advances in the field of arthroscopy have sought to enhance visual clarity and intensified the use of various types of surgical equipment, such as pressure-control devices, sealed cannulas, and electrocauterization, in an effort to reduce bleeding. ${ }^{[2]}$ Furthermore, the efficacy of the addition of EPN to the irrigation fluid on visual quality has been recently investigated in a few studies, and a significant improvement in surgeon-rated visualization during shoulder arthroscopy has been reported. ${ }^{[2]}$ Additionally, some authors have reported that TXA is effective in decreasing intraoperative bleeding during shoulder and knee replacement surgery and hemarthrosis after arthroscopic anterior cruciate ligament reconstruction. ${ }^{[8,9,11]}$ Although systemic administration of TXA has been found to be effective in obtaining better vision in shoulder arthroscopy, little is known about the impact of local administration of TXA on the surgeon's visualization during arthroscopic operations, and no study has yet investigated whether TXA is superior to EPN in providing visual clarity during arthroscopic rotator cuff repair. ${ }^{[10]}$ Therefore, the primary endpoint of the present study was the comparative results of adding TXA or EPN to the irrigation fluid for visual clarity.

The results of the present study showed that adding TXA to the irrigation fluid during the arthroscopic rotator cuff repair could provide similar visual quality to the EPN. Accordingly, our hypothesis that adding TXA to the irrigation fluid might be superior to EPN in providing visual clarity during rotator cuff tear arthroscopy was not supported by the data obtained from the present study. In another double-blind, prospective, randomized study, Avery et al. ${ }^{[6]}$ compared surgeon-rated visualization in shoulder arthroscopy using an irrigation fluid with and without EPN. The authors included 83 patients and randomized them to receive an irrigation solution with $(n=44)$ or without $(n=39)$ EPN during shoulder arthroscopy. A total of 54 of these operations were arthroscopic rotator cuff repairs, allowing a subgroup analysis, as well. The authors observed an improved visualization in the EPN group versus placebo, while comparing both only rotator cuff repairs and all procedures. In another study with a similar design to that of Avery et al., ${ }^{[6]}$ van Montfoort et al. ${ }^{[2]}$ concluded that the addition of EPN $(0.33 \mathrm{mg} / \mathrm{L})$ to the irrigation fluid conspicuously enhanced the visual clarity in most common types of arthroscopic procedures of the shoulder, which included rotator cuff repairs $(n=41)$, Bankart/SLAP repairs $(n=28)$, and subacromial procedures without rotator cuff repair $(n=32)$. While Avery et al. ${ }^{[6]}$ rated the visual clarity of the whole procedure using VAS in the immediate postoperative period, van Montfoort et al. ${ }^{[2]}$ scored the visual quality with 5-min intervals during the procedure using a subjective numeric rating scale. In our study, similar to Avery et al., ${ }^{[6]}$ the visual clarity was rated using the VAS, which is a subjective tool, in the immediate postoperative period. Of note, contrary to the aforementioned studies, our cohort of patients was larger and more homogeneous.

In the current study, we investigated secondary endpoints including TOT, total amount of irrigation fluid, the need for surgeon-demanded intravenous blood pressure therapy, and the need for an increase in pump pressure, to assess the impact of changes in the VAS on the effectiveness of the arthroscopic procedure. In consistent with the comparative results of visual clarity between the groups, no significant differences were observed in these parameters. These findings indicate that EPN and TXA provide similar surgical effectiveness, allowing similar changes in the VAS. Nevertheless, it should be underlined that the operating surgeons were experienced shoulder arthroscopic surgeons in our study and, therefore, they may have been able to perform these procedures with lower levels of image quality without extending the operating time or using more irrigation fluid.

Furthermore, potent cardiovascular drugs may cause serious cardiovascular adverse events, such as cardiac arrhythmias. ${ }^{[12-14]}$ Although adverse reactions associated with the use of an irrigation fluid with added EPN are rare, EPN-induced, potentially lethal arrhythmias during arthroscopic shoulder surgery have been reported in the literature. ${ }^{[12,13]}$ Cho et al..$^{[12]}$ reported two cases of fatal cardiovascular collapse secondary to ventricular tachycardia out of a total 
of 2,000 arthroscopic shoulder surgeries. Karns ${ }^{[13]}$ also reported a case of ventricular tachycardia requiring defibrillation during arthroscopic shoulder surgery with an irrigation fluid with added EPN in a healthy female. Mazzocca et al. ${ }^{[14]}$ presented a case of EPN-induced pulmonary edema in a 19-year-old female athlete during arthroscopic knee surgery with an irrigation fluid with added EPN. Conversely, other studies examining the adverse cardiovascular effects of an irrigation fluid with added EPN during arthroscopic surgery discovered neither arrhythmias nor obvious alterations in the MAP or heart rate. Differently from the existing literature, we attempted to compare the side effect profiles of EPN versus TXA, monitoring the MAP, electrocardiographic abnormalities, or arrhythmias during surgery. We observed no obvious cardiac abnormalities in either groups. However, three patients developed uncontrolled hypertension during the procedure in both groups and excluded from the analysis.

The most important disadvantage with the local use of TXA may be concerns about its effect on articular cartilage. In this study, the dose of TXA was chosen as $0.42 \mathrm{mg} / \mathrm{mL}$, and the mean TOT was $105.7 \pm 23.45 \mathrm{~min}$. Parker et al. ${ }^{[15]}$ described cytotoxicity in human chondrocytes and cartilage explant at a TXA concentration over $20 \mathrm{mg} / \mathrm{mL}$ at $12 \mathrm{~h}$. Also, McLean et al. ${ }^{[16]}$ showed that TXA decreased chondrocytes viability at high concentrations $(100 \mathrm{mg} / \mathrm{mL})$, as well as at low concentrations $(1 \mathrm{mg} / \mathrm{mL})$ over a longer duration $(24 \mathrm{~h})$. On the other hand, Ambra et al. ${ }^{[17]}$ found that TXA did not increase cytotoxicity when treated with porcine cartilage explants with $0 \mathrm{mg} / \mathrm{mL}$ to $4 \mathrm{mg} / \mathrm{mL}$ of TXA up to $6 \mathrm{~h}$. Sukur et al. ${ }^{[18]}$ also reported that TXA was less cytotoxic than EPN in an animal model at the doses typically used in irrigation solution. Based on these findings, we can speculate that TXA can be used in arthroscopic surgery as a safe agent at low concentrations.

Nonetheless, there are some limitations and strengths to this study. The main limitation is the subjective nature of the VAS, which is the primary endpoint measurement tool for visual clarity. Another limitation is the exclusion of two patients after randomization, which may have limited the benefits of randomization. Since this study was designed to compare TXA with EPN, there was no placebo group which can be deemed as another limitation. In addition, the absence of follow-up may have led to overlook possible side effects. On the other hand, the main strengths of our study lie in its double-blind, prospective, randomized-controlled design with a relatively large sample size.
In conclusion, our study results suggest that adding TXA to the irrigation fluid during arthroscopic rotator cuff repair provides similar visual quality to EPN, as measured by the VAS, without significant differences in the TOT and volume of irrigation fluid. Additionally, intra-articular administration of EPN or TXA does not induce adverse cardiac events.

\section{Declaration of conflicting interests}

The authors declared no conflicts of interest with respect to the authorship and/or publication of this article.

\section{Funding}

The authors received no financial support for the research and/or authorship of this article.

\section{REFERENCES}

1. Gürpınar T, Polat B, Tekin SB, Esin Polat A, Çarkçı E, Öztürkmen Y. Comparison of early clinical results and re-tear rates of transosseous-equivalent rotator cuff repairs with or without medial knots. Eklem Hastalik Cerrahisi 2019;30:193-200.

2. van Montfoort DO, van Kampen PM, Huijsmans PE. Epinephrine diluted saline-irrigation fluid in arthroscopic shoulder surgery: a significant improvement of clarity of visual field and shortening of total operation time. A randomized controlled trial. Arthroscopy 2016;32:436-44.

3. Ampat G, Bruguera J, Copeland SA. Aquaflo pump vs FMS 4 pump for shoulder arthroscopic surgery. Ann R Coll Surg Engl 1997;79:341-4.

4. Burkhart SS, Danaceau SM, Athanasiou KA. Turbulence control as a factor in improving visualization during subacromial shoulder arthroscopy. Arthroscopy 2001;17:209-12.

5. Tuijthof GJ, Dusée L, Herder JL, van Dijk CN, Pistecky PV. Behavior of arthroscopic irrigation systems. Knee Surg Sports Traumatol Arthrosc 2005;13:238-46.

6. Avery DM 3rd, Gibson BW, Carolan GF. Surgeon-rated visualization in shoulder arthroscopy: a randomized blinded controlled trial comparing irrigation fluid with and without epinephrine. Arthroscopy 2015;31:12-8.

7. Jensen KH, Werther K, Stryger V, Schultz K, Falkenberg B. Arthroscopic shoulder surgery with epinephrine saline irrigation. Arthroscopy 2001;17:578-81.

8. Gillespie R, Shishani Y, Joseph S, Streit JJ, Gobezie R. Neer Award 2015: A randomized, prospective evaluation on the effectiveness of tranexamic acid in reducing blood loss after total shoulder arthroplasty. J Shoulder Elbow Surg 2015;24:1679-84.

9. Turan S, Bingöl O. Is tranexamic acid effective on hidden blood loss in patients during total knee arthroplasty? Jt Dis Relat Surg 2020;31:488-93.

10. Liu YF, Hong CK, Hsu KL, Kuan FC, Chen Y, Yeh ML, et al. Intravenous administration of tranexamic acid significantly improved clarity of the visual field in arthroscopic shoulder surgery. A prospective, double-blind, and randomized controlled trial. Arthroscopy 2020;36:640-7.

11. Chiang ER, Chen KH, Wang ST, Ma HL, Chang MC, Liu CL, et al. Intra-articular injection of tranexamic acid reduced postoperative hemarthrosis in arthroscopic anterior cruciate ligament reconstruction: A prospective randomized study. Arthroscopy 2019;35:2127-32. 
12. Cho SH, Yi JW, Kwack YH, Park SW, Kim MK, Rhee YG. Ventricular tachycardia during arthroscopic shoulder surgery: a report of two cases. Arch Orthop Trauma Surg 2010;130:353-6.

13. Karns JL. Epinephrine-induced potentially lethal arrhythmia during arthroscopic shoulder surgery: a case report. AANA J 1999;67:419-21.

14. Mazzocca AD, Meneghini RM, Chhablani R, Badrinath SK, Cole BJ, Bush-Joseph CA. Epinephrine-induced pulmonary edema during arthroscopic knee surgery. A case report. J Bone Joint Surg [Am] 2003;85:913-5.

15. Parker JD, Lim KS, Kieser DC, Woodfield TBF, Hooper GJ. Is tranexamic acid toxic to articular cartilage when administered topically? What is the safe dose? Bone Joint J 2018;100-B:404-12.

16. McLean M, McCall K, Smith IDM, Blyth M, Kitson SM, Crowe LAN, et al. Tranexamic acid toxicity in human periarticular tissues. Bone Joint Res 2019;8:11-8.

17. Ambra LF, de Girolamo L, Niu W, Phan A, Spector M, Gomoll AH. No effect of topical application of tranexamic acid on articular cartilage. Knee Surg Sports Traumatol Arthrosc 2019;27:931-5.

18. Sukur E, Kucukdurmaz F. Comparison of Cytotoxic Effects of Intra-Articular Use of Tranexamic Acid versus Epinephrine on Rat Cartilage. Med Sci Monit 2018;24:1166-70. 